\title{
Tensiones y desafíos de la participación ciudadana en materia ambiental ${ }^{* * * * * * * *}$
}

\author{
Challenges and tensions of public \\ participation in environmental matters
}

\section{RESUMEN}

Este trabajo reflexiona sobre las particularidades que tiene la participación en materia ambiental. Las distintas maneras como los ordenamientos jurídicos la recogen y regulan fundamenta una cierta distancia teórica con el tratamiento general que se da a la participación en la gestión pública. No obstante, se indaga también en otros factores de distanciamiento con el debate general sobre participación, lo que involucra la existencia de variables que tensionan la participación ambiental, como los conceptos de riesgo, deliberación, justicia ambiental y acceso a la justicia. Lo anterior conduce a plantear la necesidad de construir un tratamiento teórico diferenciado, que integre miradas interdisciplinarias (derecho, ciencias políticas, sociología, entre otras) en orden a entender y regular adecuadamente el asunto.

PALABRAS CLAVE

Participación, medio ambiente, gestión pública.

Abogado, magíster y doctor en Derecho. Director de Investigación de la Facultad de Derecho de la Universidad de Chile y profesor asociado del Departamento de Derecho Público de la misma misma (Santiago de Chile, Chile). Contacto: fsoto@ derecho.uchile.cl

ORCID ID: 0000-0002-9358-5217.

** Abogado, magíster en Regulación y doctor (c) en Derecho. Investigador del Centro de Regulación y Competencia (RegCom) de la Facultad de Derecho y del Instituto Milenio para la Investigación de Imperfecciones de Mercado y Políticas Públicas (MIPP) de la Universidad de Chile (Santiago de Chile, Chile). Contacto: ecosta@derecho.uchile.cl ORCID ID: 0000-0003-3928-8622.

**** Recibido el 13 de marzo de 2018, aprobado el 11 de junio de 2019.

Para citar el artículo: Soto Barrientos F. y Costa Cordella, E. Tensiones y desafíos de la participación ciudadana en materia ambiental. En Revista Derecho del Estado, Universidad Externado de Colombia. N. . 44, septiembre-diciembre de 2019, pp. 227-255.

Dor: https://doi.org/10.18601/01229893.n44.09

***** Este trabajo forma parte del proyecto de investigación Fondecyt Regular $2017 \mathrm{n}$. ${ }^{\circ}$ 1170767, "Procedimiento de Creación de Normas: Planes de Prevención y Descontaminación Ambiental". 
ABSTRACT

This paper is a reflection over the singularities of public participation in environmental issues. The way in which the law deals with it in practice shows differences between public participation in this area, and public participation in general, that could imply a theoretical difference. Moreover, this difference can also be studied from the variables that justify public participation in environmental matters, such as risk management, environmental justice, deliberation and access to justice. Because of these differences, this article believes that public participation in environmental issues should be treated different from a theoretical point of view, integrating disciplines such as political science, sociology and law, to understand and regulate accurately the subject.

KEYWORDS

Public Participation, Environmental Law, Public Management.

SUMARIO

Introducción. 1. La participación ciudadana en la gestión pública. 2. Participación en la gestión pública y accountability. 3. Participación ciudadana en materia ambiental. 4. Tensiones en la participación en materia ambiental. 4.1. Participación en materia ambiental y riesgos. 4.2. Participación en materia ambiental y deliberación. 4.3. Participación en materia ambiental y justicia ambiental. 4.4. Participación en materia ambiental y acceso a la justicia. Conclusiones. Referencias.

\section{INTRODUCCIÓN}

En un escenario de creciente valorización del aporte que significa la participación ciudadana en la gestión pública en Occidente, la práctica existente muestra cierta tendencia a la especialización. Hoy la realidad de la participación ciudadana dista de ser uniforme. Por otra parte, una de las materias en que la participación se ha desarrollado de manera acelerada es la ambiental, generando una serie de desafíos que son propios de esa área y que la singularizan.

Así, mientras se puede observar que el desarrollo doctrinal de la participación en la gestión pública se vincula principalmente con el principio democrático en la gestión pública y como instrumento de control político, pareciera ser que en el caso de la participación en materia ambiental su desarrollo ha estado relacionado con varios otros ámbitos que exigen un tratamiento diferenciado.

En materia ambiental, además, como se verá, el modelo predominante de comprensión de la participación ciudadana proviene de la Declaración 
de Río de 1992, donde se entrelazaron tres mecanismos -luego reconocidos como derechos de acceso- en la toma de decisiones ambientales, esto es, el acceso a la información, la participación ciudadana y el acceso a la justicia. Dicha realidad dista de la forma en que la participación en la gestión pública ha sido comprendida.

Esta observación desafía una conceptualización general que entiende que entre la participación en materia ambiental y la participación en la gestión pública existe una relación de género a especie, y lleva a preguntar sobre los desafíos que doctrinariamente debe sortear la participación en materia ambiental si quiere comprenderse a sí misma, otorgarse funciones claras y, finalmente, cumplir sus objetivos.

El presente artículo pretende mostrar de manera general los desafíos que esta distinción comporta, no singularizando las tensiones en ningún sistema jurídico nacional en particular ni en las respuestas formuladas por dichos ordenamientos, sino desde una visión global del asunto. Lo anterior debido al carácter global del problema ambiental y de la crisis de la democracia representativa, pero asimismo a la evolución paralela de los ordenamientos ambientales nacionales ${ }^{1}$.

\section{LA PARTICIPACIÓN CIUDADANA EN LA GESTIÓN PÚBLICA}

El punto de partida del presente trabajo es la participación en la gestión pública y la forma en que su tratamiento en la doctrina relevante ha evolucionado. De especial importancia en este acápite resulta la comprensión de los orígenes y fines de la participación en la gestión pública, pues ella permitirá la comparación con la participación en materia ambiental.

La progresiva falta de compromiso político es un mal endémico de las democracias contemporáneas. Si bien la existencia de un sistema representativo constituye el supuesto básico para el buen funcionamiento de dichas democracias, la experiencia comparada justifica la incorporación de otras formas de participación ciudadana, alternativas a la elección de autoridades, con el objetivo de revertir esta tendencia a la apatía, de complementar el funcionamiento de las instituciones y, de esta manera, profundizar en el desarrollo de la institucionalidad democrática² .

Con todo, los mecanismos institucionales elaborados a partir de la segunda mitad del siglo xx en Europa han repercutido básicamente en la configura-

1 Se ha excluido del análisis la participación de los pueblos indígenas, por cuanto entendemos que esta tiene sus propias particularidades que tensionan incluso a la participación ambiental y que por lo tanto son un campo de estudio que, si bien presenta superposiciones con la participación en la gestión pública y la participación ambiental, es diferente.

2 Luciani, M. La formazione delle leggi. T. I, 2 Art. 75, Il referendum abrogativo. En Commentario della Costituzione, fondato da G. Branca e continuato da A. Pizzorusso, Bolonia y Roma: Zanichelli e Il Foro Italiano, 2005. 
ción de fórmulas alternativas de decisión legislativa y constitucional. Esto se traduce, en la práctica, en la configuración de una serie de mecanismos de democracia directa o participativa, como son distintos tipos de referendos o iniciativas legislativas populares que hoy se reconocen en las constituciones de Europa y Latinoamérica preferentemente ${ }^{3}$.

No obstante, es solo a partir de las últimas décadas que el análisis de la participación se ha centrado en la gestión pública, en la medida en que las decisiones sustantivas de interés público se han trasladado desde el clásico espacio deliberativo del parlamento a organismos administrativos o agencias de diversa denominación. El foco cambió, de concentrar iniciativas para mejorar la dinámica de la sociedad sobre el Estado (toma de decisiones legislativas o constitucionales), al impacto del Estado sobre la sociedad, expresado en la implementación de políticas públicas. Lo anterior en cuanto es la Administración quien tiene actualmente mayor presencia en la configuración del espacio social inmediato de los ciudadanos.

Así, la representación quedaría reducida a un simple mecanismos de legitimación con respecto a quién gobierna, pero no en cuanto a qué y cómo lo hace, elementos confiados a claves autónomas de racionalidad y tecnocracia definidas por el Gobierno y la Administración ${ }^{4}$. Esto genera una consecuencia inevitable: el Congreso decide entregar a agencias públicas, sujetas a determinados tipos de procedimientos, la adopción de las reglas del juego sustantivas, lo que genera inevitables problemas de legitimidad para la definición de sus contenidos ${ }^{5}$. Esto corresponde, de algún modo, a la denominada etapa reflexiva del derecho, en donde se produce una orientación procedimental de estructuras que, ordenadas relacionalmente, generan condiciones legítimas para las decisiones ${ }^{6}$.

Esa falta de legitimidad en la toma de decisiones públicas es en parte la que genera interés por los mecanismos de participación ciudadana, siendo que se reconoce en ellos la potencialidad de superar esa barrera y revestir de legitimidad a las decisiones una vez que han sido objeto de los mecanismos de participación.

Así las cosas, se han articulado una serie de instituciones donde la participación ciudadana opera con anterioridad a la toma de decisiones efectuada por organismos administrativos (esta instancia, en términos generales, tienen por objetivo justamente aportar nuevos y mejores antecedentes al órgano público responsable). En estos casos, la intervención de la ciudadanía se

3 Sото, F. El desafío de la participación. Estudio comparado sobre el referéndum y la iniciativa legislativa popular en América Latina y Europa. Santiago: LoM, 2013.

4 Porras Nadales, A. Representación y democracia avanzada. En Cuadernos y Debates. 1994. Madrid: CEC.

5 Habermas, J. Más allá del Estado nacional. México: FCE, 1998.

6 Teubner, G. Substantive and reflexive elements in modern law. En Law and Society Review. 1983, vol. 17, 239-285. 
concreta en un nivel básico: consultiva, informativa y contralora. Por lo general, la participación ciudadana en este modelo no es decisoria, con la salvedad de la modalidad de democracia participativa en que los ciudadanos tienen facultades co-decisorias, en relación, por ejemplo, con experiencias particulares de participación orgánica -los ciudadanos actúan al interior del órgano público- o en relación con la constitución de foros de participación donde pueden adoptar decisiones, lo que regularmente no sucede más allá del ámbito local. Es la autoridad pública la que se reserva la potestad decisoria, si bien otorga realce, por su conexión con el principio democrático, a los procedimientos que incluyan la opinión de la ciudadanía?.

En ese eje decisional de la autoridad se instala la participación, como uno de los factores que contribuye a balancear las variables democrática y técnica. En este sentido, la OCDE ha señalado que la evaluación que hacen los ciudadanos respecto de sus gobiernos está enfocada principalmente en dos rendimientos: el de las políticas públicas (técnico) y el democrático. De acuerdo con el organismo internacional, el rendimiento de las políticas públicas estaría marcado por la posibilidad de entregar resultados positivos a la ciudadanía; mientras que el rendimiento democrático estaría vinculado a la medida en que la toma de decisiones se encuentre en línea con los principios de la democracia. La participación ciudadana ayudaría a reforzar ambos, el primero mejorando el resultado, y el segundo mejorando el proceso ${ }^{8}$.

La distinción realizada por la OCDE resulta interesante, en el sentido de que la evaluación de las políticas públicas podría hacerse tanto desde su procedimiento de creación como desde sus eventuales efectos. En cuanto a su procedimiento, el rendimiento democrático sería la variable principal, lo que equivaldría a evaluar la manera en que esa toma de decisiones por parte del Estado está efectivamente vinculada con el mandato del pueblo, cuestión abordable en parte por los procedimientos de participación ciudadana. En cuanto a sus efectos, lo que pareciera importar en este análisis es que la política pública finalmente decidida tenga la potencialidad de abordar los objetivos que se plantea y de resolver los asuntos para los cuales fue creada. Esta segunda visión, entonces, más propia de la eficacia y la eficiencia, debiera ser balanceada con la primera, para efectos de obtener algo que pudiéramos llamar buenas políticas públicas.

Es por esto que las recomendaciones de política pública de la OCDE han venido enfatizando el rol y la importancia de los mecanismos de participa-

7 Callahan, K. Elements of effective governance: Measurement, accountability and participation. En Public Administration and Public Policy. 126, 2007 y Castellá Andreu, J. M. Los derechos constitucionales de participación política en la Administración Pública. En Estudios constitucionales y políticos. Barcelona: Cedecs, 2001.

8 OECD. Focus on Citizens: Public Engagement for Better Policy and Services. 2009, 23. [En línea]. Disponible en: http://www20.iadb.org/intal/catalogo/pe/2009/03785.pdf [Consultado el 31 de agosto de 2017]. 
ción ciudadana en la gestión pública, y empujando a sus países miembros a adoptar más medidas en este sentido. Así por ejemplo y en específico en materia ambiental, la OCDE ha recomendado en 2016 a Chile "perfeccionar el proceso de realización de evaluaciones de impacto ambiental, con el fin de asegurar que se tomen seriamente en consideración proyectos alternativos, se garantice la participación ciudadana en las etapas preliminares y se otorgue más atención a los efectos ambientales potenciales, especialmente en los ecosistemas" . Agregando que ha habido mejoras desde 2005, no obstante lo cual "las disposiciones vigentes sobre participación de la ciudadanía en estas evaluaciones no contienen disposiciones que garanticen la adecuada consideración de proyectos alternativos o la minimización del impacto ambiental potencial, lo que puede dar origen a conflictos ambientales y sociales" ${ }^{10}$.

Ahora bien, existe asimismo cierto nivel de crítica a esta visión de la participación ciudadana, y en ese sentido Dryzek considera, por ejemplo, que los mecanismos de participación (discursive design) son creados por quien tiene el poder y por lo tanto no buscan desafiarlo, cuestión que se traduciría en un menor rendimiento de la herramienta. Ello, sin embargo, es matizado por el propio autor, al considerar que de todas maneras dichos mecanismos abren un espacio en que puede producirse este desafío ${ }^{11}$.

Como señala Fiorino, además, "enfrentamos un dilema participativo, donde las expectativas de las personas sobre su capacidad de influir en las decisiones, no tienen un correlato con la realidad de las instituciones políticas" ${ }^{12}$. Vale decir que mientras la democracia promete que las decisiones obedecerán a un sentir mayoritario, la realidad es que una parte importante de las decisiones se ha vuelto más bien técnica y no recoge efectivamente los resultados de los procesos de participación.

La discusión planteada es interesante, en cuanto constituye uno de los cuestionamientos a la participación ciudadana que resultan más problemáticos. Por un lado, la participación genera legitimidad de las políticas públicas, pero por el otro, cuando dicha participación es llevada adelante con el único propósito de generar esa legitimidad este efecto se ve mermado. La consecución de mayor legitimidad es, en definitiva, el resultado de una participación que se realiza bajo ciertos estándares de calidad, que a su vez están definidos en diferentes instrumentos. No se puede decir que la legitimidad sea un resultado automático. La crítica de Dryzek se desarrolla sobre la misma idea:

9 OCDE, CEPAL. Evaluación del desempeño ambiental de Chile. 2016, 18. [En línea]. Disponible en: http://repositorio.cepal.org/bitstream/handle/11362/40308/S1600413_es.pdf [Consultado el 31 de agosto de 2017].

10 Ibíd.

11 DryzeK, J. Ecology and discursive democracy: Beyond liberal capitalism and the administrative State. En Capitalism Nature Socialism. 1992, 3, 2, 18-42, 36.

12 Fiorino, D. J. Environmental risk and democratic process: A critical review. En Colum. J. Envtl.L. 1989, 14, 503. 
mientras el mecanismo esté diseñado por quien detenta el poder para evitar ser cuestionado, el efecto esperado de la participación no se producirá ni para los participantes ni para el Estado.

En la actualidad se cuenta con un amplio abanico de mecanismos, como las consultas directas a la ciudadanía, adicionadas con otros mecanismos participativos como la audiencia pública, la participación en el procedimiento administrativo, el derecho de petición, de información administrativa, de la defensa del interesado o de la intervención de órganos consultivos, los que han impactado en la manera de concebir el buen gobierno ${ }^{13}$. Este nuevo paradigma es reforzado por la literatura actual, la que ha identificado que la participación ciudadana en políticas públicas promueve la eficacia política y estimula el debate público; que se dan efectos positivos de estos procesos en los gobiernos, fortaleciendo la capacidad de recepción de las demandas ciudadanas ${ }^{14}$; y que se produce un efecto integrador y legitimador que, incluso, generaría importantes niveles de satisfacción en las personas que participan ${ }^{15}$.

\section{PARTICIPACIÓN EN LA GESTIÓN PÚBLICA Y ACCOUNTABILITY}

Un tratamiento complementario al tradicional de la participación ciudadana en la gestión pública es el que se le ha dado en su maridaje con el concepto de accountability, elaborado en el ámbito académico anglosajón.

En una visión tradicional, este concepto envuelve los mecanismos políticos y legales con que se revisa el uso del poder público y la manera como este se legitima, cuestión que se ha ido modificando y ampliando en virtud de fenómenos similares a los comentados en la primera sección de este trabajo, especialmente con base en una demanda de mayor participación ${ }^{16}$. El debate sobre accountability estuvo normalmente ligado a los procedimientos de toma de decisión y a la justicia de los mismos. Hoy, además, parece haber avanzado más allá del poder de las agencias reguladoras y estar enfocado en los regímenes regulatorios en su conjunto, lo que supone una preocupación no solo por la decisión en sí misma, sino por la relación entre los diferentes actores que intervienen en el proceso de toma de decisión.

Una de las perspectivas en este debate ha sido precisamente la del empoderamiento ciudadano, que supone la necesidad de accountability directo de los tomadores de decisiones, mediante procedimientos de participación

13 Pernice, I. Multilevel constitutionalism in the European Union. En European Law Review. 2002, 27, 5, 511-529.

14 Lupia, A. y Matsusaka, J. G. Direct democracy: New approaches to old questions. En Annual Review of Political Science. 2004, 7, 463-482.

15 Frey, B. y Stutzer, A. Happiness and Economics. Princeton y Oxford: Princeton University Press, 2002.

16 Baldwing, R.; CAVE, M. y Lodge, M. Understanding Regulation. Theory, Strategy and Practice. 2. ${ }^{a}$ ed. Oxford: Oxford University Press, 2012, 338-339. 
ciudadana. Estos mecanismos serían necesarios, en contraste con la posibilidad de confiar en decisiones técnicas. "[E]n una interpretación extensiva, el accountability, de acuerdo a esta visión, se refiere al mayor involucramiento de los ciudadanos" 17 .

Desde esta mirada, entonces, el concepto de accountability incluiría toda una serie de demandas de la sociedad civil a la esfera de la acción pública: disponibilidad y acceso a la información por parte de los ciudadanos; control de resultados a través de una adecuada cuantificación evaluativa de los efectos de las políticas públicas; transparencia en las decisiones públicas; imputación de la responsabilidad pública. En consecuencia, el concepto de accountability operaría en dos fases diferenciadas: la dimensión procesal, afectando al itinerario de la gestión pública como producto de un debate social, $\mathrm{y}$, por otro lado, estableciendo mecanismos efectivos de control a posteriori $i^{18}$.

Como señala Callahan, tanto el accountability como la participación ciudadana son partes cruciales de una gobernanza efectiva; así, cuando coexisten ambos podemos considerar que hay accountability democrática ${ }^{19}$. En este sentido, si bien la autora recoge varias definiciones distintas de la literatura sobre accountability, llama la atención la que ella asume, en relación con lo que significaría para los ciudadanos: "el accountability del sector público es la obligación que tienen las autoridades de explicar de manera pública, completa y suficiente la manera como llevan a cabo o fallan en llevar a cabo responsabilidades que afectan al público de manera importante" 20 .

Se hace evidente la relación de este concepto con la participación ciudadana a nivel procesal, pues en los procesos de participación es donde precisamente las personas pueden exigir el cumplimiento de esta obligación de reporte, siendo además que la participación obliga a la apertura de ciertos antecedentes que también constituyen parte del accountability.

Callahan, siguiendo a Romzek y Dubnick, explica además las cuatro dimensiones que tiene esta rendición de cuentas: (i) Burocrática, entendida como la rendición de cuentas jerárquica de los funcionarios públicos; (ii) Legal, que implica la revisión de organismos externos como los tribunales; (iii) Profesional, referida al adecuado uso de su lex artis y a la evaluación de pares, y (iv) Política, que importa la satisfacción de determinadas expectativas de un grupo relevante, típicamente, los electores ${ }^{21}$.

Desde el mundo de la participación en la gestión pública, parecería que al menos el accountability político y el accountability profesional tienen un

17 Ibíd., 352.

18 HunOLD, C. Pluralism, and democracy: Toward a deliberative theory of bureaucratic accountability. En Governance, 2001, 14, 151-167, 110.

19 Callahan, K. Elements of effective governance: Measurement, accountability and participation. En Public Administration and Public Policy. 126, 2007, 108.

$20 \quad$ Ibíd., 217.

21 Ibíd., 114-116. 
lugar entre las funciones que la participación cumple, especialmente tomando en cuenta las obligaciones de respuesta. Seguidamente, el accountability legal también podrá estar involucrado, pues la participación de alguna manera también fuerza a los tomadores de decisiones a abrir y explicar más profundamente las motivaciones de una decisión, cuestión que luego puede ser revisada por organismos contralores y tribunales.

Explicadas estas dos formas de comprender la participación ciudadana en la gestión pública, lo que nos interesa saber ahora es cómo se ha entendido la participación en materia ambiental, y en qué medida recoge estas conceptualizaciones o las desafía.

\section{PARTICIPACIÓN CIUDADANA EN MATERIA AMBIENTAL}

La participación ciudadana en materia ambiental y su regulación han ido de la mano del vertiginoso protagonismo que tienen en las sociedades contemporáneas los problemas de contaminación. Estados Unidos y el Reino Unido fueron los primeros en establecer mecanismos de participación pública en materia ambiental, dando cuenta de una tendencia que se extendería posteriormente a todo el mundo ${ }^{22}$. Desde la perspectiva legislativa comparada, los sistemas institucionales han transitado desde un reconocimiento del acceso a la información, la presentación de alegaciones y comentarios, a la articulación de mesas de negociación, cuyo acceso queda limitado a determinados grupos de presión, como asociaciones empresariales u organizaciones no gubernamentales, hasta llegar a fórmulas más deliberativas y ciudadanas, donde se establecen espacios de diálogos abiertos para la definición de decisiones públicas en materia de medio ambiente ${ }^{23}$.

Pese a que la Declaración de Estocolmo de 1972 hace las primeras menciones a la necesidad de incorporar la participación ciudadana a la resolución de conflictos ambientales ${ }^{24}$, es en la Declaración de Río de 2012 donde se fijan los actuales estándares participativos en materia ambiental. Así, más de 120 países han adoptado disposiciones legales que aseguran la participación ciudadana en las evaluaciones de planes de impacto ambiental ${ }^{25}$.

22 Van Bekhoven, J. Public participation as a general principle in international environmental law: Its current. En National Taiwan University Law Review. 2016, vol. 11, 2, 219-271.

23 Richardson, B. y Razzaque, J. Public participation in environmental decision-making. En Environmental Law for Sustainability. 2006, 165-194.

24 Declaración de Estocolmo de 1972, punto 7 del Preámbulo: "Para llegar a esta meta será menester que ciudadanos y comunidades, empresas e instituciones, en todos los planos, acepten las responsabilidades que les incumben y que todos ellos participen equitativamente en la labor común".

25 Banisar, D.; PARMar, S.; De Silva, L. y Excell, C. Moving from principles to rights: Rio 2012 and access to information, public participation, and justice. En Sustainable Development Law \& Policy. 12, n. ${ }^{\circ}$ 3, 2012, 8-14. 
Dentro de la lógica de la cuestión ambiental y en especial del derecho ambiental, la demanda por participación es uno de los pilares del movimiento ambientalista $^{26}$, y ello se refleja en que esté siempre presente en las discusiones de política pública ambiental a nivel nacional e internacional. La piedra angular pareciera ser el Principio 10 de la Declaración de Río, pero además esto se ha traducido en la celebración del Convenio de Aarhus a nivele europeo, las "Directrices para la elaboración de legislación nacional sobre el acceso a la información, la participación del público y el acceso a la justicia en asuntos ambientales" (Directrices de Bali) ${ }^{27}$ y la negociación de un tratado especial sobre el Principio 10 a nivel latinoamericano ${ }^{28}$.

El hecho es que, en materia ambiental, la participación ciudadana constituye uno de los pilares fundamentales sobre los que se ha construido el derecho de esta rama, manteniendo a la participación elevada al nivel de principio y a la vez como parte integrante de los conceptos basales de la disciplina, como son la justicia ambiental y el derecho a vivir en un medio ambiente sano, adecuado o libre de contaminación.

Ferrer identifica esto con lo que llama la fase participativa, que sería uno de los estratos con los que se ha ido construyendo el derecho ambiental ${ }^{29}$. En este estrato se reconocería la necesidad de construcción conjunta de las políticas públicas, entre el Estado y los ciudadanos, además de la fuerte inclusión de estos últimos en la toma de decisiones del primero ${ }^{30}$.

De manera más general, Fraga reconoce en la participación uno de los contenidos procesales del derecho subjetivo a un medio ambiente adecuado en el derecho español ${ }^{31}$. En una línea similar, Ballesteros-Pinillas considera que puede verse que la participación en materia ambiental, bien sea como "postulado funcional" o como principio, es un criterio que debe guiar a los

26 En este sentido, por ejemplo, Dryzek et al. consideran que la participación es una de las bases de lo que él llama modernización ecológica fuerte. Los autores distinguen entre los diferentes discursos que podrían modificar las instituciones para hacerlas más protectoras del medio ambiente y posiciona a la modernización ecológica como una de las que tienen más posibilidades de ser incorporadas en la lógica actual de los Estados, por ser menos cuestionadora de estructuras esenciales. De ahí que la manera en que efectivamente los Estados han ido incorporando lógicas más ambientalistas vaya en ese sentido, en el que la participación ciudadana, como dijimos, es un elemento primordial. Dryzek, J. S.; Downes, D.; Hunold, C.; SchlosberG, D. y Hernes, H. K. Green States and Social Movements: Environmentalism in the United States, United Kingdom, Germany, and Norway. Oxford: oup, 2003, 169.

27 Véase: http://www.mma.gob.cl/1304/articles-55240_DirectricesBaliAcceso_2010.pdf

28 Véase: https://negociacionp10.cepal.org

29 Ferrer reconoce 5 estratos, que organiza de manera cronológica, aunque no necesariamente sucesiva: (i) la fase represiva, (ii) la fase preventiva, (iii) la fase participativa, (iv) las técnicas de mercado y la internacionalización de los costes, y (v) las técnicas integrales.

30 ReAl Ferrer, G. La construcción del derecho ambiental. En Novos Estudos Jurídicos. 2013, 18, 3, 347-368. DOI: 10.14210/nej.v18n3, p. 356-357.

31 Fraga JoRDANO, J. El derecho a disfrutar de un medio ambiente adecuado: elementos para su articulación expansiva. En Medio Ambiente y Derecho. 1998. 
Estados en su deber de protección del medio ambiente y, consecuentemente, a los poderes públicos que de estos derivan, incluido el judicial ${ }^{32}$.

Con todo, se mantiene la interrogante de si este marco legal, de cooperación entre la esfera pública y la sociedad civil, de transparencia de la información y de control de la gestión pública, está a la altura de los desafíos que suponen las actuales democracias avanzadas. Es aquí donde los crecientes conflictos ambientales desafían la naturaleza e intensidad de las fórmulas participativas existentes ${ }^{33}$. Esto debido a que la credibilidad de los gobiernos y de las empresas ha sido sistemáticamente cuestionada por una ciudadanía cada vez más reflexiva y consiente de los impactos sociales y ambientales del desarrollo económico ${ }^{34}$. Por lo tanto, para algunos autores resulta evidente que los modelos de gestión pública participativa se han visto superados, y que se debe avanzar en fórmulas que mejoren el vínculo con los ciudadanos ${ }^{35}$.

Un debate que viene diferenciando los ordenamientos nacionales que regulan la participación en materia ambiental tiene que ver con si se regula como principio, como derecho o como un simple mecanismo que va de la mano de las garantías que protegen el medio ambiente.

En primer término, se tiene la comprensión de la participación como un principio, cuestión particularmente relevante en materia ambiental, donde se le ha reconocido con mayor fuerza. En segundo lugar, la participación es reconocida como un derecho, y allí es donde en específico se posiciona la participación en la gestión pública, a propósito de su reconocimiento legal en diversas legislaciones e incluso en tratados internacionales como la Carta Iberoamericana de Participación en la Gestión Pública. Por último, la participación es analizada desde la dimensión de los mecanismos de participación, donde encontramos, por supuesto, un sinnúmero de posibilidades para concretar la participación, desde audiencias públicas hasta comentarios online, pasando por consejos consultivos, proyectos de cogobierno y planes piloto, por señalar algunos ejemplos. Es en esta última dimensión, además, en la que suele estar centrada una de las discusiones más comunes respecto de la participación ciudadana, que es la referida al nivel de incidencia que dichos mecanismos tienen y debiesen tener en la toma de decisiones por parte de la autoridad.

32 Ballesteros Pinilla, G. La participación en asuntos ambientales y su tutela en el Convenio de Aarhus. En Vniversitas. N. ${ }^{\circ} 121,2010,19-48,26$.

33 BÁRCENA, I. ¿Democracia participativa + desarrollo sostenible $=$ democracia ambiental? Preguntas y cuestiones para la democratización ecológica, cit. en IBARRA, P. y AHEDo, I. (eds.). Democracia participativa y desarrollo humano. Madrid: Dykinson, 2008.

34 Bеск, U. La sociedad del riesgo. Hacia una nueva modernidad. Barcelona: Paidós, 2002.

35 Benn, S. y Jones, R. The role of symbolic capital in stakeholder disputes: Decisionmaking concerning intractable wastes. En Journal of Environmental Management. 2009, 90, 1593-1604. 
En general, las regulaciones en materia de participación ambiental se han desarrollado como consecuencia del Principio 10 de la Declaración de Río de 1992, que fue el que dio pie a la creación de provisiones específicas de acceso a la información, participación y acceso a la justicia en materia ambiental. La expansión y relevancia de este modelo se evidencia, por ejemplo, en el hecho de que el principal índice que mide la democracia en materia ambiental en el mundo lo haga precisamente desde esos tres indicadores ${ }^{36}$.

Ahora bien, el Principio 10 en sí mismo no conceptualiza el acceso a la información, la participación y el acceso a la justicia como derechos, sino que reclama la necesidad de que existan mecanismos que los recojan. En ese sentido, por ejemplo, un interesante trabajo de Mahuerhofer catastró la existencia de estos mecanismos a nivel global, sean o no reconocidos como derechos. El citado autor parte de la base de que la expansión de estos mecanismos tiene que ver con un trabajo que se ha construido sobre la base del Principio 10 de la Declaración de Río, y además aboga por su reconocimiento mediante mecanismos legales obligatorios ${ }^{37}$.

Esta diferencia es notada también por Okubo, quien, refiriéndose a la regulación de la participación ciudadana en materia ambiental en Japón, si bien parte de la base de que su consagración ha tenido que ver con el Principio 10, de todas maneras hace un distingo entre la forma en que se ha consagrado en otros países (principalmente en Europa), a lo que llama un enfoque basado en derecho, y la manera en que se ha hecho en Japón, que identifica como un enfoque con base voluntaria ${ }^{38}$.

Pareciera que parte importante de la diferencia que se observa entre los modelos de participación guarda relación con los instrumentos normativos internacionales que han guiado o fundamentado la construcción de las regulaciones en materia de participación ambiental. En este escenario son especialmente importantes la Declaración de Río de 1992 y la Convención de Aarhus, acordada en 1998. Esta última, particularmente, establece en su artículo primero el derecho al acceso a la información, la participación ciudadana en la toma de decisiones y el acceso a la justicia en materia ambiental, como garantías que contribuyen a la protección del derecho de las personas y de las generaciones futuras a vivir en un medio ambiente adecuado.

Desde el paradigma de la Convención de Aarhus se plantea la mayor parte de la doctrina continental, tanto europea como latinoamericana, que ha tratado estos derechos de acceso. Pareciera que, en general, desde este modelo

36 Véase: http://www.environmentaldemocracyindex.org/about/background_and_methodology

37 Mauerhofer, V. Public participation in environmental matters: Compendium, challenges and chances globally. En Land Use Policy. 2016, n. ${ }^{\circ}$ 52, 481-491.

38 Окиво, N. The development of the Japanese legal system for public participation in land use and environmental matters. En Land Use Policy. N. 52, 492-500. 
ninguno de los tres derechos puede ser entendido ni ejercido sin relacionarlo de alguna forma con los demás; la información es base para poder participar o accionar judicialmente, y el acceso a la justicia es la única garantía de que los otros dos derechos de acceso se cumplan, y con ello sus fines.

Como señala Torres, "existe consenso a nivel internacional de que la Convención de Aarhus, al ser vinculante, es el instrumento que más lejos ha llegado en cuanto a promover los derechos de acceso. Ha sido calificada como la iniciativa más ambiciosa en el terreno de la democracia ambiental que se haya emprendido jamás bajo los auspicios de las Naciones Unidas. A la fecha ninguna otra región ha avanzado en el desarrollo de un instrumento legal vinculante similar a la Convención de Aarhus" 39 .

El que observamos como avance principal de la Convención de Aarhus es la consolidación del modelo del Principio 10 de la Declaración de Río, mediante la incorporación de un lenguaje de derechos en el que estos mecanismos elevan su categoría jurídica y se sitúan en el centro del derecho ambiental. Probablemente este ascenso tenga relación con la necesidad de una respuesta a los conflictos ambientales, ante la imposibilidad de dar solución técnica y de estándares a la problemática ambiental. Se prefiere un modo procesal, que es más sencillo de acordar en arenas más amplias, postergando discusiones más de fondo para que sean abordadas en otras instancias.

Lee y Abbott analizan las motivaciones del Convenio de Aarhus y notan que su objetivo final sería mejorar las condiciones de protección de la naturaleza, cuestión para la cual se apoya y confía en la participación de los ciudadanos, entendiendo que teóricamente dicha participación es la que logrará mejorar la protección del medio ambiente ${ }^{40}$.

Así las cosas, entre estos derechos procedimentales, sin duda, la participación ocupa el lugar central, siendo asistida por los otros dos, en el entendido de que es la participación la que en realidad ayuda a mejorar las decisiones y a superar el déficit democrático en materia ambiental ${ }^{41}$.

En la misma línea del Convenio de Aarhus es que se ha venido negociando desde 2012, en Latinoamérica, un convenio para la aplicación del Principio 10 de la Declaración de Río. Dicho proceso comenzó con la "Declaración sobre la aplicación del Principio 10 de la Declaración de Río sobre el Me-

39 CEPAL. Acceso a la información, participación y justicia en temas ambientales en América Latina y el Caribe: situación actual, perspectivas y ejemplos de buenas prácticas. En Medio Ambiente y Desarrollo. 2013, 15.

40 Lee, M. у Аввот, C. The usual suspects? Public participation under the Aarhus Convention. En Modern Law Review. Vol. 66, n. ${ }^{\circ}$ 1, 2003, 86.

41 Ballesteros Pinilla, analizando el Convenio de Aarhus, reconoce que los niveles de participación que este impone a las partes se diferencian según el tipo de espacio en que se lleve a cabo dicha participación. En ese sentido, la obligación sería más fuerte cuando se está en el nivel de actividades específicas, mientras que no lo sería tanto cuando se discuten políticas públicas o medidas reglamentarias. Véase Ballesteros PinIlla. La participación en asuntos ambientales y su tutela en el Convenio de Aarhus, cit., 28-31. 
dio Ambiente y el Desarrollo", suscrita por 10 países de la región y en que manifiestan:

Reconocemos y subrayamos que los derechos de acceso a la información, participación y justicia en materia ambiental son esenciales para la promoción del desarrollo sostenible, de la democracia y de un medio ambiente sano, y aportan múltiples beneficios tales como: contribuir a tomar mejores decisiones y aplicarlas más eficazmente; involucrar al público respecto de los problemas ambientales; aportar a la rendición de cuentas y la transparencia en la gestión pública; y facilitar un cambio en los patrones de producción y consumo ${ }^{42}$.

Al día de hoy, en dicha negociación confluyen 24 países de la región ${ }^{43}$.

Vale la pena detenerse a considerar cómo la negociación de un acuerdo internacional sobre participación en materia ambiental se enmarca inmediatamente en la lógica del Principio 10 de la Declaración de Río, como modelo ideal de participación. En efecto, ni siquiera se hace mención a las lógicas de la participación en la gestión pública. La hegemonía del modelo de derechos de acceso viene refrendada, por ejemplo, en el estudio comparado de Torres para la CEPAL sobre democracia ambiental en Latinoamérica, que al buscar las normas sobre participación en esta materia también lo hace de inmediato desde ese paradigma, siendo al final un estudio relativo al "Acceso a la información, participación y justicia en temas ambientales en América Latina y el Caribe"44.

Incluso, el modelo del Principio 10 ha llegado a constitucionalizarse. Este proceso, como reconocen May y Daly, obedecería al ánimo de permitir un ulterior avance de la protección del medio ambiente ${ }^{45}$. En particular, la participación en cuestiones ambientales estaba contemplada, a 2015, en las constituciones de Brasil, Colombia, Ecuador, Eritrea, Etiopía, Finlandia, Francia, Islandia, Kosovo, Polonia, Tailandia y Zambia ${ }^{46}$.

Ahora bien, aun cuando el Principio 10 y su modelo sean hegemónicos en Europa y Latinoamérica, este no es el único modelo que es posible detectar. En primer lugar, para el derecho ambiental internacional, el principio de participación no parece ser de mayor trascendencia, y así, revisados los principales manuales de la materia, este principio no se encuentra tratado,

42 Véase: http://www.un.org/ga/search/view_doc.asp?symbol=A/CONF.216/13\&referer=/ english/\&Lang=S

43 Véase: https://negociacionp10.cepal.org/7/es/noticias/paises-la-region-concluyennueva-ronda-negociacion-acuerdo-derechos-acceso-materias

44 CEPAL. Acceso a la información, participación y justicia en temas ambientales en América Latina y el Caribe: situación actual, perspectivas y ejemplos de buenas prácticas. Serie Medio Ambiente y Desarrollo n. ${ }^{\circ}$ 151. Santiago de Chile, 2013.

45 MaY R., J.y DaILY, E. Global Environmental Constitucionalism. Nueva York: Cambridge University Press, 2015, 77.

46 Ibíd., 373-374. 
mientras que otros, como los de prevención, precaución y desarrollo sostenible, son explorados en todos ellos ${ }^{47}$.

Asimismo, en el derecho estadounidense tampoco pareciera que la participación sea abordada como un principio del derecho ambiental, ni tampoco como un tema central de la garantía de un medio ambiente sano. Así, por ejemplo, reputados manuales de derecho ambiental de dicho país no la consideran dentro de los temas principales ni la abordan como una cuestión especial ${ }^{48}$. En algunos casos excepcionales se aborda la participación ciudadana, pero como un mecanismo dentro de otros procedimientos de la National Environmental Protection Act (NEPA), y sin darle una especial relevancia, ni menos tratarla como derecho ${ }^{49}$.

En lo que se refiere al manual de participación ciudadana de la agencia ambiental de Estados Unidos (la EPA), este tampoco se refiere a la participación como un derecho ni la relaciona con la garantía. La manera en que la trata es haciendo variadas referencias a sus funciones, especialmente a aquellas relacionadas con la mejora de la decisión del organismo público, su legitimidad y efectividad potencial ${ }^{50}$. Pareciera entonces que, en el ámbito estadounidense, la participación ambiental se parece más a la participación en la gestión pública.

Por su parte, la Environmental Law Worldwide Alliance (ELAw) realizó un extenso análisis comparado de la participación ciudadana en materia ambiental en Estados Unidos, Canadá y México. En lo que acá interesa, dicho estudio explica cómo la participación ciudadana en Estados Unidos en materia ambiental se diferencia claramente según si se está ante procesos asociados a regulaciones o a proyectos. En cuanto a las regulaciones, la norma proviene en primera instancia de la ley de procedimiento administrativo, que impone la necesidad de "notificar y comentar" a los potenciales interesados cuándo se va a modificar una regulación en general, incluidas las regulaciones ambientales. Luego, cada ley con alcances ambientales ha considerado a su vez una manera particular de llevar adelante la participación ciudadana cuando se estén dictando otras regulaciones del área en que la ley aplica $(v \cdot g r .$, la

47 Birnie, P.; Boyle, A. y Redgwell, C. International Law \& the Environment. 3. a ed. Nueva York: Oxford University Press, 2009; Hunter, D.; SAlzMAn, J. y ZaElKe, D. International Environmental Law and Policy. New York: Foundation Press, 1998; SANDS, P. Principles of International Environmental Law. 2. ${ }^{\text {a }}$ ed. Cambridge University Press, 2003; De SAdELEer, N. Environmental Principles: From Political Slogans to Legal Rules. Nueva York: Oxford University Press, 2002.

48 Revesz L., R. Foundations of Environmental Law and Policy. Nueva York: Foundation Press, 1997; Percival V., R.; Schroeder H., C. y Miller S., A. Environmental regulation. En Law, Science, and Policy. 4. ${ }^{a}$ ed. 1996; Malone A., L. Environmental Law. 2. ${ }^{a}$ ed. The Emanuel Law Outlines Series. Nueva York, 2007; KubaSeK K., N. y Silverman S., G. Environmental Law. 6. ${ }^{\mathrm{a}}$ ed. New Jersey, 2008.

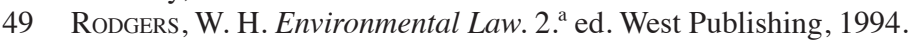

50 Véase: https://www.epa.gov/sites/production/files/2015-09/documents/intro_08.pdf 
ley de aire limpio, la ley de agua limpia y la ley de control de sustancias tóxicas). Por último, la Ley Nacional de Políticas Ambientales, que regula la evaluación ambiental de proyectos, reconoce de manera más específica la participación ciudadana y los modos en que esta debe llevarse a cabo en dicho procedimiento. Esto no altera de forma significativa la comprensión de la participación como un mecanismo y un paso dentro del procedimiento ${ }^{51}$.

Tanto el acceso a la información como el acceso a la revisión judicial de las decisiones de las agencias reguladoras están garantizados también por la ley de procedimiento administrativo.

Con todo, pareciera que el sistema estadounidense de participación ciudadana en materia ambiental está más basado en su propia tradición de derecho administrativo que en la comprensión internacional de la participación, el acceso a la información y el acceso a la justicia como una trenza de derechos que en su faz procedimental hacen posible la protección del derecho a vivir en un medio ambiente sano, que sería el modelo predominante en Europa y en Latinoamérica.

\section{TENSIONES EN LA PARTICIPACIÓN EN MATERIA AMBIENTAL}

El hecho de que la participación ciudadana en materia ambiental no sea fácilmente tratable como una especie del género de la participación ciudadana en la gestión pública se relaciona con que existen variables que tensionan los conceptos de esta última, cuando se alude a cuestiones ambientales. Dichas variables no solo imponen un desafío a la participación en materia ambiental en sí misma, en cuanto a su necesidad de abordarlas, sino que también importan una tensión entre este tipo de participación y la participación en la gestión pública.

En el presente acápite se recogen las variables que aparecen como más fundamentales en provocar estas tensiones, que son la incorporación de los conceptos de riesgos, deliberación, justicia ambiental y acceso a la justicia.

\subsection{Participación en materia ambiental y riesgos}

Una de las cuestiones centrales del derecho ambiental y las regulaciones ambientales es la gestión de los riesgos provocados, ya sea por proyectos que tienen impactos ambientales, ya sea por procesos que tienen esos impactos. Tanto en la toma de decisiones generales de regulación como en la toma de decisiones particulares de proyectos, la gestión del riesgo está siempre presente como una de las variables que es preciso considerar.

51 Véase: https://www.elaw.org/es/content/gu\%C3\%AD-de-participaci\%C3\%B3nciudadana-en-materia-ambiental-de-m\%C3\% A9xico-estados-unidos-y-canad\%C3\% A1espa\% $3 \% \mathrm{~B} 1 \mathrm{o}$ 
Esteve Pardo considera la gestión del riesgo como uno de los paradigmas del derecho ambiental, expresando que "[e]l derecho al Medio Ambiente es un derecho de regulación y gestión de riesgos que se explica y encuentra su sentido en ese modelo de sociedad posmoderna en la que estamos instalados y que se ha dado en llamar la sociedad del riesgo" 52 . En la misma línea, Astorga señala que "[v]ivimos en una sociedad del riesgo, en la cual el rol de las normas ambientales corresponde fundamentalmente el establecimiento de niveles de seguridad, los que tienen por objeto evitar la exposición del daño"53.

Vale decir que para una parte de la doctrina, el derecho ambiental en general se dedica principalmente a la gestión de los riesgos de las actividades con impactos ambientales. Esto se torna especialmente interesante en nuestro análisis, pues, como hemos señalado, la participación es uno de los principios del derecho ambiental, y por lo tanto debería estar conversando de alguna forma con esta necesidad de gobernar los riesgos.

Según Beck, la dinámica ambiental da cuenta de un proceso de "individualización", que inevitablemente llevará a una toma de decisiones potencialmente más inclusiva. Esto porque los sistemas tradicionales de autoridad, ya sean políticos, administrativos, legales o científicos, han facilitado y legitimado procesos de modernización asociados a una subestimación institucionalizada del "riesgo". En este contexto, la insatisfacción con la congestión de los procesos institucionales tradicionales derivará en la formación de redes temporales y de múltiples actores que funcionan como espacios descentralizados, autodeterminados, dinámicos y flexibles para la toma de decisiones. Estas nuevas estructuras políticas, concluye Beck, no solo serán más inclusivas sino que permitirán la entrada de nuevas formas de conocimiento. Los círculos previamente cerrados de grupos de expertos se enfrentan a conflictos y desafíos, lo que llevará al desarrollo de lo que Beck denomina "conocimiento no lineal" 54 .

Es común encontrar críticas relacionadas con la poca importancia que tiene la ciudadanía en la toma de decisiones sobre el riesgo, puesta en frente de los expertos técnicos y científicos, que son los que realmente serán quienes tomen las decisiones ${ }^{55}$. En la misma lógica, Rosanvallon, analizando a Beck, señala que "[1]a sociedad del riesgo por su propia naturaleza es cautelosa del futuro, pero los ciudadanos siguen obligados a poner su confianza en los científicos, porque no pueden sopesar los asuntos relevantes sin la ayuda de especialistas" ${ }^{\prime 6}$.

52 Esteve Pardo, J. Derecho del medio ambiente. 3. a ed. Madrid: Marcial Pons, 2014, 14.

53 Astorga, E. Derecho ambiental chileno. Santiago: Lexis Nexis, 2006, 32.

54 Bеск, U. La sociedad del riesgo. Hacia una nueva modernidad. Barcelona: Paidós, 2002.

55 Fiorino, D. J. Environmental risk and democratic process: A critical review. En Colum. J. Envtl. L. 1989, 14, 503.

56 Rosanvallon, P. Counter-democracy Policy in the Age of Distrust. Cambridge, 2009, 9. 
El hecho de que la participación sea dejada de lado a la hora de gobernar los riesgos puede explicarse en la fundamentación doctrinaria que han tenido autores como Sunstein, quien releva el papel de los científicos para la toma de decisiones relativas al riesgo, por sobre las percepciones que tienen las personas. El argumento de Sunstein no es extremista y considera que hay de todas maneras una cuestión democrática de por medio para la toma de decisiones, pero cree que ella solo puede operar cuando no está cargada de sesgos propios de las personas al percibir los riesgos ${ }^{57}$.

En ese sentido, Moya, en referencia a Sunstein, señala:

Lo anterior se traduce en que las personas comunes y corrientes tienen una determinada percepción del riesgo, en la que no hay una relación entre aquellas situaciones que la gente percibe como más peligrosas y las que efectivamente lo son. El pensamiento cotidiano comete errores al valorar el riesgo y produce apreciaciones distorsionadas. Muchas veces, la percepción exagerada se funda en la mayor relevancia mediática de unos sucesos o en la subjetividad de cada persona $^{58}$.

La apreciación de estos autores pretende que en cierto nivel de certeza científica, las personas nos preocuparíamos más de riesgos que son improbables que de otros que son mucho más probables e igualmente catastróficos. Pero ello no parece suficiente para abstraer de la ciudadanía la decisión sobre qué riesgos deben abordarse y cómo. Primero, por la evidente tensión democrática que esa disociación supone, pero además por una cuestión práctica que tiene que ver con cuál es el objetivo de la gestión de riesgos. La percepción que los ciudadanos tienen sobre el riesgo, y por lo tanto el temor y el impacto de ese temor en sus vidas, es también una afectación objetiva, como podrían ser los daños que la materialización de ese riesgo supondría. Dicha afectación no puede ser descartada por intrascendente sin más.

Todo lo anterior es lo que Fiorino ha llamado dilema participatorio en las políticas de riesgo: "El aumento de la complejidad de los asuntos abordados por la política pública del gobierno, en particular la confianza en los datos y el método científico, refuerza otras tendencias que disminuyen la influencia individual. Ahora los científicos y la élite técnica juegan un rol influyente en la toma de decisiones sociales" 59 .

De ahí que se hayan desarrollado enfoques que hablan de "gobernanza ambiental", la cual supone políticas, estructuras y procesos que permitan el control y la coordinación de la toma de decisiones de las partes interesadas

57 Sunstein, C. Riesgo y razón. Buenos Aires: Katz, 2006, 157-159.

58 Moya, F. El principio de precaución. Santiago: Legal Publishing, 2014, 45.

59 FIORINO. Environmental risk and democratic process, cit., 532. 
y que facilitará la definición de un conjunto de valores compartidos entre los actores afectados por decisiones ambientales ${ }^{60}$.

\subsection{Participación en materia ambiental y deliberación}

Desde la teoría de la democracia deliberativa y de la argumentación también se particularizan las potencialidades participativas de los conflictos ambientales $^{61}$, si bien los teóricos de la democracia deliberativa hacen extensiva la necesidad de la deliberación a un sinfín de temáticas de interés público, inclusive incorporando el concepto de democracia deliberativa, que pugna con otros como el de democracia radical o participativa ${ }^{62}$.

Destaca, en esta óptica, la perspectiva de Dryzek ${ }^{63}$, el cual llega a proponer el concepto de "democracia ecológica", donde sistematiza metodologías para garantizar una toma de decisiones más racional desde el punto de vista ambiental. El mismo autor, en su análisis de los discursos de la política ambiental, señala que uno de los que mayormente han empujado cambios ha sido el del pragmatismo democrático, con base en el cual -sin perjuicio de las críticas que formula Dryzek- se han relevado los ideales de la participación ciudadana en materia ambiental, como una manera de incorporar democracia en las decisiones de la autoridad y también de hacer que dichas decisiones sean cada vez más protectoras del medio ambiente ${ }^{64}$.

Para algunos, las dimensiones particulares que reviste el conflicto ambiental, que suponen un grado adicional de sensibilidad participativa, impactan de manera profunda en las bases que sustentan los diseños institucionales. Así, Jaría ${ }^{65}$ considera que los desafíos medioambientales hacen necesaria una reformulación del constitucionalismo actual, el que está sustentado en un individualismo posesivo que permite el despliegue del proceso de acumulación capitalista.

En este sentido, Lee y Abbot destacan como una de las diferencias importantes de la participación en materia ambiental el hecho de que se requiere por parte de los ciudadanos no solo un aporte en la creación de las regula-

60 Benn, S. y Jones, R. The role of symbolic capital in stakeholder disputes: Decisionmaking concerning intractable wastes. En Journal of Environmental Management. 2009, 90, 1593-1604.

61 ECKERSLEY, R. He big chill: The wTo and multilateral environmental agreements. En Global Environmental Politics. Vol. 4, 2004, 24-50.

62 Elster, J. La democracia deliberativa. Barcelona: Gedisa, 2001.

63 Dryzer, J. Deliberative Democracy and Beyond, Liberals, Critics, Contestations. Oxford Political Theory, 2004; DryzeK, J. The Politics of the Earth: Environmental Discourses. Oxford University Press, 2005; DRYZEK, J. y GoOdIN, R. Deliberative impacts: the macro-political uptake of mini-publics. En Politics \& Society. 2006, 34, 2, 219-244.

64 DRYZEK. The Politics of the Earth, cit.

65 Jaría, J. El constitucionalismo de la escasez: derechos, justicia y sostenibilidad. En Revista Aranzadi de Derecho Ambiental. 2015, n. ${ }^{\circ}$ 30, 295-349. 
ciones, sino también en la solución concreta de los problemas ambientales. Lo anterior supondría niveles de involucramiento de los ciudadanos que van más allá de la mera posibilidad de influir en las decisiones, pues son parte tanto de las causas de los problemas como de sus soluciones ${ }^{66}$. Las mismas autoras, sin embargo, en un artículo diferente, dan cuenta de que claramente las prácticas de participación ciudadana en materia ambiental no alcanzan el ideal deliberativo, siendo que las típicas consultas públicas que se hacen en estos casos no van más allá del modelo típico de decisión de experto, siendo la participación solo un input más a la decisión ${ }^{67}$.

Por último, la tensión en cuestión ha sido estudiada por Smith, quien considera que el movimiento ambiental es uno en el que conviven una pluralidad de miradas y valores, los cuales además deben interactuar con otros valores sociales, en orden a lograr incrementar la protección del medio ambiente. De acuerdo al autor, la manera en que ello podría ser logrado es precisamente mediante los mecanismos deliberativos, siendo por eso que desde los movimientos ambientalistas se perseguiría tener estos mecanismos, que permitirían una interacción en un plano de igualdad entre los valores del movimiento y otros valores sociales ${ }^{68}$. La decisión tomada de manera deliberativa, señala Smith, tendría la virtud de generar flujos de información más abiertos, que permitirían la incorporación de los afectados por una decisión. En esto, el autor compara la democracia deliberativa con la democracia liberal, haciendo hincapié en las falencias de esta última para lograr lo que Dryzek llama la racionalidad ecológica, que es precisamente la toma de decisiones exenta de sesgos y más comprensiva de las variables, a la que se llega mediante la deliberación ${ }^{69}$.

En definitiva, la relación entre la protección ambiental y la deliberación vendría dada por la pretensión que existe en la regulación y la política ambiental de incorporar de mayor y mejor manera a las personas. Esa pretensión provendría de las propias características de la regulación ambiental, incluyendo la estrecha relación de las personas con el territorio y la presión por incorporar nuevos valores en la definición de políticas públicas, de modo que ellas tiendan a la conservación y protección del medio ambiente.

\subsection{Participación en materia ambiental y justicia ambiental}

El concepto de justicia ambiental tiene múltiples acepciones, todas ellas concentradas en cuestiones distributivas relacionadas con el medio ambiente.

66 Lee, M. у Аввот, C. The usual suspects? Public participation under the Aarhus Convention. En Modern Law Review. Vol. 66, n. ${ }^{\circ}$ 1, 2003, 83.

67 Ibíd., 86.

68 Smith, Graham. Deliberative Democracy and the Environment. Abingdon: Routledge, 2003.

69 Ibíd. 
Una de dichas acepciones es la que mira la distribución de poder a propósito de la toma de decisiones ambientales, considerando que las personas que son afectadas por una determinada decisión debieran tener la posibilidad de influir en ella de manera significativa ${ }^{70}$.

Entonces, la participación ciudadana en materia ambiental, a la vez que es considerada en sí como una expresión de la justicia ambiental, también es un vehículo para la consecución de la misma en lo sustantivo. Respecto de lo primero, el propio concepto de justicia ambiental parte de una realidad en la que la inadecuada distribución de cargas ambientales se suma a otras formas de discriminación en contra de las comunidades afroamericanas y pobres. Por lo mismo, y como señala Schlosberg, la participación ha sido desde el comienzo una de las cuestiones clave para el movimiento ambiental, y se ha mantenido como una demanda permanente del movimiento ambiental y de las comunidades ${ }^{71}$.

Sobre este punto, Hervé define a la justicia ambiental en relación con la participación como justicia ambiental en el ámbito político. De acuerdo a esta autora, "esta segunda dimensión [de la justicia ambiental] sostiene, por lo tanto, que no debe analizarse la justicia solo en relación de sus consecuencias, sino que debe también considerar sus causas"72.

En relación con lo segundo y como decíamos, la justicia ambiental abarca diferentes ámbitos de distribución. Comienza en el movimiento ambiental norteamericano como un concepto de justicia distributiva en sentido clásico u horizontal, pero avanza rápidamente en dos variables. Desde la horizontalidad, se amplía para abarcar no solamente a los miembros de una comunidad política determinada, sino a otras (o todas las) formas de vida que habitan un territorio. En el sentido vertical, se nutre del concepto de justicia intergeneracional para abarcar también a las generaciones futuras.

Así entonces, podemos observar cómo la relación entre justicia ambiental y participación ciudadana tensiona a esta última en al menos dos sentidos cruciales. El primero, referido a que la participación debe tanto cubrir las causas de las injusticias ambientales como ser un espacio que permita revertirlas. El segundo, relacionado con que además la hace responsable, en parte, de lograr una mejor distribución de las cargas y beneficios ambientales, cuestión que podría abarcar desde los miembros actuales de una comunidad política hasta todos los seres vivos actuales y los que vendrán.

70 Hervé Espejo, D. Justicia ambiental y recursos naturales. Valparaíso: Ediciones Universitarias de Valparaíso PUCv, 2015, 59.

71 Schlosberg, D. Defining Environmental Justice. Nueva York: Oxford University Press, 2007, 65-68.

72 HeRvé ESPEJO. Justicia ambiental y recursos naturales, cit., 43. 


\subsection{Participación en materia ambiental y acceso a la justicia}

A pesar del incremento en la regulación de la participación ambiental, vemos cómo, cada vez más, los conflictos ambientales no necesariamente se canalizan por esta vía sino por la vía judicial ${ }^{73}$.

Por lo demás, y como vimos anteriormente, la participación ciudadana en materia ambiental es conceptualizada en su relación con el acceso a la justicia en la materia, a propósito de la trenza que realiza el Principio 10 de la Declaración de Río y sus desarrollos posteriores.

Se hace necesario, entonces, al analizar la participación ambiental, tener en cuenta esta otra dimensión que corre generalmente paralela. Según Reyes, el activismo ambiental y la judicialización de los conflictos acompañan los procesos de participación, muchas veces, estigmatizándolos como insuficientes y no vinculantes. La mayoría de las veces estos conflictos se ven tensionados por una dinámica de oposición y controversia fundada en la percepción de incompatibilidad en torno al uso, acceso y/o preservación del medio ambiente y sus recursos.

La complejidad y las características de estos conflictos, que habitualmente no suponen definiciones puramente ambientales, sino que afectan dimensiones sociales, económicas, culturales, políticas e institucionales, han generado una gran diversidad de nuevos actores. Así vemos cómo, junto a las personas naturales directamente afectadas, se articulan agrupaciones locales, organismos no gubernamentales, empresas e instituciones públicas ${ }^{74}$.

No es tan claro lo señalado por Reyes, en cuanto ella adopta una perspectiva que mira a la participación y a la judicialización como cuestiones alternativas, e incluso cree que los procesos judiciales iniciados por los ciudadanos tienden a denostar a la participación ciudadana. Hay sin embargo un error conceptual en este sentido, provocado por la confusión de los fines de la participación y de los procesos judiciales. Si bien ambos comparten ciertos objetivos (como ser mecanismos de accountability), la verdad es que la participación ciudadana no es un espacio de mediación para solucionar conflictos, menos todavía si esos conflictos son entre privados ${ }^{75}$.

73 Tate, C. N. y Vallinder, T. (eds.). The Global Expansion of Judicial Power. New York University Press, 1995.

74 ReYes, F. Las Cortes y los conflictos socioambientales: ¿quo vadis poder judicial? En Anuario de Derecho Público, Universidad Diego Portales. 2014, 293-316.

75 Esa confusión es en todo caso el producto claro de una configuración normativa particular. Como la misma autora señala, algunos conflictos ambientales son provocados por una visión diferente del uso del territorio entre una actividad determinada y una comunidad o parte de ella. Ese conflicto es en principio entre esas partes, pero tiende a ser canalizado frente al tomador de desiciones, abogando cada una de las partes por su interés frente a esa entidad. Sin embargo, una vez que se ha tomado la desición es posible que se produzca litigio entre la parte que no vio satisfecho su interés y el tomador de desiciones, a quien típicamente se demandará por no haber considerado un determinado conjunto de argumentos que lo habrían conducido a tomar 
El acceso a la justicia y la participación ciudadana constituyen, junto al acceso a la información, una trenza que permite a los ciudadanos enfrentarse de alguna manera al poder del Estado y los titulares de proyectos, tal como es reconocido en el Principio 10 de la Declaración de Río y teorizado en la manera antes descrita. Aunque la doctrina ha tendido a ser escéptica respecto de la efectividad de la participación ciudadana en el terreno judicial ${ }^{76}$, recientes estudios demuestran lo contrario. Brinks y Gauri ${ }^{77}$, al comparar la intervención judicial en materia de derechos económicos y sociales, destacan el impulso que tienen estos conflictos en lo que ellos denominan procesos de legalización.

Así, los tribunales, abogados y los diversos actores sociales generarían toda una suerte de base conceptual y de lenguaje que finalmente termina incidiendo en las regulaciones y políticas públicas adoptadas. La legalización es, en consecuencia, un efecto indirecto y continuo generado por la participación en conflictos judiciales, donde litigios repetitivos y coordinados resultan clave para que este proceso se produzca adecuadamente. De ahí la importancia de la articulación de organizaciones de la sociedad civil en el resguardo de derechos fundamentales ${ }^{78}$.

En esta misma línea, Rosanvallon ve en la judicialización de las políticas públicas uno de los factores de constitución de la contra-democracia (entendida no como una negación de la democracia, sino como una especie de contrapeso). Para el autor, la búsqueda de soluciones judiciales en áreas de política pública estaría marcada por la disminución de la responsividad de los gobiernos y por lo tanto por un aumento en las demandas de rendición de cuentas ${ }^{79}$.

Desde la teoría de la participación ciudadana en la gestión pública, entenderíamos que una de las funciones que esta cumple es la que podríamos denominar "de control", que se construye sobre la idea de que mediante la participación ciudadana es posible moldear y controlar el poder del Estado y el uso que los funcionarios hacen del mismo. Esta función también ha sido

una decisión contraria. Cuando quienes reclaman ante la decisión del ente son los ciudadanos, efectivamente es muy posible que se denoste el proceso de participación, en el entendido de que no logró el objetivo por ellos buscado. Cuando la decisión es denegar un permiso o imponer una multa, se produce el mismo fenómeno pero en un espacio diferente, siendo el titular de una actividad el que reclamará frente a la decisión, probablemente denostando el proceso respectivo por no haberse cumplido su expectativa. Así entonces, no parece tan claro que la relación entre participación y litigación sea la que se señala en el citado artículo, e incluso podríamos llegar a concluir que en determinadas circunstancias la propia litigación es una forma de participación, independiente de que en ella se critique el procedimiento específico de participación previo a la toma de una decisión. Véase ibíd.

76 Damaska, M. Las caras de la justicia. Análisis comparado del proceso. Santiago: Jurídica de Chile, 2000.

77 Brinks D. y Gauri, V. Courting Social Justice: Judicial Enforcement of Social and Economic Rights in the Developing World. Cambridge University Press, 2008.

78 Ibíd.

79 Rosanvallon, P. Counter-democracy Policy in the Age of Distrust. Cambridge, 2009, 16. 
llamada función defensiva ${ }^{80} \mathrm{o}$ de monitoreo $^{81}$. En ese entendido, la función de control podría encontrar su máxima expresión en la posibilidad de que un tribunal, como tercero imparcial encargado de la resolución de conflictos, conozca y resuelva casos en los que los procesos de participación ciudadana hayan tenido ciertas dificultades.

En materia ambiental, esta función de control es mucho más fuerte y en muchos casos enfocada en el interés general de la protección del medio ambiente como un bien colectivo. Por ello, además de que participación y acceso a la justicia están profundamente relacionados, sería posible, bajo ciertas condiciones, entender que la judicialización en sí misma podría ser un tipo de participación o es parte de la participación.

\section{CONCLUSIONES}

En el presente artículo hemos analizado los conceptos de participación ciudadana en la gestión pública y de participación ciudadana en materia ambiental. En un primer momento, cabría pensar que el segundo tipo de participación es parte del primero, en cuanto parecería que las decisiones que se toman en materia ambiental estarían incluidas dentro de la gestión pública. Además, analizadas ambas materias, parecería que las funciones que vienen a cumplir, en cuanto espacio de incorporación de variables democráticas, información y control, dentro de la toma de decisiones públicas son también muy similares o coincidentes.

Lo anterior, sin embargo, es solo parcialmente cierto. Mientras la doctrina de participación en la gestión pública resulta ilustrativa para comprender la participación en materia ambiental, no alcanza a cubrir todas las variables que esta última tiene. Por una parte, esto es así por el hecho de que el derecho ambiental es la disciplina en que más se ha tratado y debatido el tema, siendo que la participación en esta área ha tomado un camino propio y ha evolucionado de manera independiente, llegando incluso a existir tratados internacionales y negociaciones internacionales que se ocupan de la participación exclusivamente en materia ambiental.

Pero además de la cuestión concreta, referida a la evolución paralela de la participación ciudadana en la gestión pública y en materia ambiental, pudimos observar cómo en esta última existen cuestiones de fondo que tensionan su relación con el concepto general de participación, que pareciera no incorporar las mismas realidades que la participación en materia ambiental.

80 CAstellá Andreu, J. M. Los derechos constitucionales de participación política en la Administración Pública. En Estudios constitucionales y políticos. Barcelona: Cedecs, 2001.

81 Lee, M. у Аввот, C. The usual suspects? Public participation under the Aarhus Convention. En Modern Law Review. Vol. 66, n. ${ }^{\circ}$ 1, 2003. 
En primer término, la participación en materia ambiental tiene una relación especial con la distribución de riesgos, a propósito de los niveles tolerables de estos y la estrecha relación entre los riesgos y las cuestiones ambientales. En segundo lugar, la participación en materia ambiental debe hacerse cargo de los conceptos de justicia ambiental, donde pudimos ver que la propia participación juega un rol preponderante en la consecución de la justicia, cuando una de las dimensiones de la misma es la justicia ambiental en sentido político. En seguida, también la participación está fuertemente vinculada al derecho a vivir en un medio ambiente sano o adecuado y se ha entendido que es parte del mismo.

Una tercera tensión tiene que ver con la deliberación, en cuanto en materia ambiental esta sería aún más necesaria y urgente que en otras materias, dado especialmente el hecho de que la solución de los problemas ambientales requiere de una información que está descentralizada y también de acciones colectivas, todo lo cual pasa por procesos de participación. Por último, la estrecha relación entre participación en materia ambiental y acceso a la justicia importa comprender que muchas veces la propia judicialización de una materia ambiental es una extensión del proceso de participación, cumpliendo con los mismos fines que se atribuyen a dichos procesos.

Es interesante destacar, además, que del estudio del tratamiento de la participación ciudadana en materia ambiental es posible desprender modelos distintos para su comprensión en el mundo occidental. Uno de ellos, predominante en Latinoamérica y Europa, parte del Principio 10 de la Declaración de Río de 1992 y ve a la participación como un derecho que está encadenado con los derechos de acceso a la información y acceso a la justicia. Esta visión ha sido además reforzada por la Convención de Aarhus de 1998. Un segundo modelo sería el estadounidense, que comprende a la participación como uno de los pasos procedimentales dentro de las normas específicas que se refieren al medio ambiente, y que releva fuertemente su importancia a partir de sus funciones, en lugar de hacerlo a partir de su naturaleza de derecho.

Con todo, el presente artículo avanza en la comprensión de la participación ciudadana en materia ambiental, acotando en parte sus límites, al menos en lo que se refiere a su relación con la participación ciudadana en la gestión pública. Entendemos que en este esfuerzo, la utilización de herramientas transdisciplinarias que al menos integren derecho, ciencia política y sociología, se hace muy necesario para lograr una adecuada comprensión del fenómeno y luego intentar regularlo de manera que cumpla con sus objetivos. 


\section{REFERENCIAS}

Astorga, E. Derecho ambiental chileno. Santiago: Lexis Nexis, 2006.

Baldwing, R.; Cave, M. y Lodge, M. Understanding Regulation. Theory, Strategy and Practice. 2. ${ }^{\text {a }}$ ed. Oxford: Oxford University Press, 2012.

Ballesteros Pinilla, G. La participación en asuntos ambientales y su tutela en el Convenio de Aarhus. En Vniversitas. N. ${ }^{\circ} 121,2010,19-48$.

Banisar, D.; Parmar, S. De Silva, L. y Excell, C. Moving from Principles to Rights: Rio 2012 and Access to Information, Public Participation, and Justice. En Sustainable Development Law \& Policy. 12, n. ${ }^{\circ}$ 3, 2012, 8-14.

Beck, U. La sociedad del riesgo. Hacia una nueva modernidad. Barcelona: Paidós, 2002.

Beck, U. y Beck-Gernsheim, E. Individualization Institutionalized Individualism and its Social and Political Consequences. Londres: Sage, 2002.

Benn, S. y Jones, R. The role of symbolic capital in stakeholder disputes: Decision-making concerning intractable wastes. En Journal of Environmental Management. 2009, 90, 1593-1604.

Birnie, P.; Boyle, A. y Redgwell, C. International Law \& the Environment. 3. a ed. Nueva York: Oxford University Press, 2009.

Brinks, D. y Gauri, V. Courting Social Justice: Judicial Enforcement of Social and Economic Rights in the Developing World. Cambridge University Press, 2008.

Callahan, K. Elements of effective governance: Measurement, accountability and participation. En Public Administration and Public Policy. 126, 2007.

Castellá Andreu, J. M. Los derechos constitucionales de participación política en la Administración Pública. En Estudios constitucionales y políticos. Barcelona: Cedecs, 2001.

CEPAL. Acceso a la información, participación y justicia en temas ambientales en América Latina y el Caribe: situación actual, perspectivas y ejemplos de buenas prácticas. Serie Medio Ambiente y Desarrollo n. ${ }^{\circ}$ 151. Santiago de Chile, 2013.

Damaska, M. Las caras de la justicia. Análisis comparado del proceso. Santiago: Jurídica de Chile, 2000.

De SAdeleEr, N. Environmental Principles: From Political Slogans to Legal Rules. Nueva York: Oxford University Press, 2002.

Dryzer, J.Deliberative Democracy and Beyond.Liberals, Critics, Contestations. Oxford Political Theory, 2004.

DRYZEK, J. Ecology and discursive democracy: Beyond liberal capitalism and the administrative State. En Capitalism Nature Socialism. 1992, 3, 2, 18-42.

Dryzer, J. The Politics of the Earth: Environmental Discourses. Oxford University Press, 2005. 
Dryzer J. S.; Downes, D.; Hunold, C.; Schlosberg, D. y Hernes, H. K. Green States and Social Movements: Environmentalism in the United States, United Kingdom, Germany, and Norway. Oxford: OUP, 2003.

Dryzer, J. y Goodin, R. Deliberative impacts: the macro-political uptake of mini-publics. En Politics \& Society. 2006, 34, 2, 219-244.

ECKERSLEY, R. He big chill: The wTO and multilateral environmental agreements. En Global Environmental Politics. Vol. 4, 2004, 24-50.

Elster, J. La democracia deliberativa. Barcelona: Gedisa, 2001.

Esteve Pardo, J. Derecho del medio ambiente. 3. a ed. Madrid: Marcial Pons, 2014.

Fiorino, D. J. Environmental risk and democratic process: A critical review. En Colum. J. Envtl. L. 1989, 14, 503.

FRAGA JoRDANo, J. El derecho a disfrutar de un medio ambiente adecuado: elementos para su articulación expansiva. En Medio Ambiente y Derecho. 1998.

Frey, B. y Stutzer, A., Happiness and Economics. Princeton y Oxford: Princeton University Press, 2002.

Habermas, J. Más allá del Estado nacional. México: FCe, 1998.

Hervé EsPeJo, D. Justicia ambiental y recursos naturales. Valparaíso: Ediciones Universitarias de Valparaíso Pucv, 2015.

Hunold, C. Pluralism, and Democracy: Toward a Deliberative Theory of Bureaucratic Accountability. En Governance. 2001, 14, 151-167.

Hunter, D.; Salzman, J. y Zaelke, D. International Environmental Law and Policy. Nueva York: Foundation Press, 1998.

Ibarra, P. y Ahedo, I. (eds.). Democracia participativa y desarrollo humano. Madrid: Dykinson, 2008.

JARIA, J. El constitucionalismo de la escasez: derechos, justicia y sostenibilidad. En Revista Aranzadi de Derecho Ambiental. 2015, n. ${ }^{\circ}$ 30, 295-349.

Kubasek K., N. y Silverman S., G. Environmental Law. 6. a ed. New Jersey, 2008.

Lee, M. у Аввот, C. The usual suspects? Public participation under the Aarhus Convention. En Modern Law Review. Vol. 66, n. ${ }^{\circ}$ 1, 2003.

LuCiani, M. La formazione delle leggi. T. I, 2, Art. 75, Il referendum abrogativo. En Commentario della Costituzione, fondato da G. Branca e continuato da A. Pizzorusso, Bolonia y Roma, Zanichelli e Il Foro Italiano, 2005.

Lupia, A. y Matsusaka, J. G. Direct democracy: New approaches to old questions. En Annual Review of Political Science. 2004, 7, 463-482.

Malone A., L. Environmental Law. 2. ${ }^{\text {a }}$ ed. The Emanuel Law Outlines Series. Nueva York, 2007. 
Martí, J. L. La república deliberativa. Barcelona: Marcial Pons, 2006.

Mauerhofer, V. Public participation in environmental matters: Compendium, challenges and chances globally. En Land Use Policy. N..$^{\circ}$ 52, 2016, 481-491.

May R., J. y Daily, E. Global Environmental Constitucionalism. Nueva York: Cambridge University Press, 2015.

Moya, F. El principio de precaución. Santiago: Legal Publishing, 2014.

oCDE, CEPAL. Evaluación del Desempeño Ambiental de Chile. 2016. [En línea]. Disponible en: http://repositorio.cepal.org/bitstream/handle/11362/40308/S1600413_es.pdf [Consultado el 31 de agosto de 2017].

oecD. Focus on Citizens: Public Engagement for Better Policy and Services. 2009. [En línea]. Disponible en: http://www20.iadb.org/intal/catalogo/pe/2009/03785.pdf [Consultado el 31 de agosto de 2017].

Окиво, N. The development of the Japanese legal system for public participation in land use and environmental matters. En Land Use Policy. N. ${ }^{\circ}$ 52, 492-500.

Percival V., R; Schroeder H., C. y Miller S., A. Environmental Regulation. Law, Science, and Policy. 4. ed. 1996.

PERNICE, I. Multilevel constitutionalism in the European Union. En European Law Review. $2002,27,5,511-529$.

Porras Nadales, A. Representación y democracia avanzada. En Cuadernos y Debates. 1994. Madrid: CEC.

Real Ferrer, G. La construcción del derecho ambiental. En Novos Estudos Jurídicos. 2013, 18, 3, 347-368. Dor:10.14210/nej.v18n3. p. 347-368.

Revesz L., R. Foundations of Environmental Law and Policy. New York: Foundation Press, 1997.

Reyes, F. Las Cortes y los conflictos socioambientales: ¿quo vadis poder judicial? En Anuario de Derecho Público, Universidad Diego Portales. 2014, 293-316.

Richardson, B. y Razzaque, J. Public participation in environmental decision-making. En Environmental Law for Sustainability. 2006, pp. 165-194.

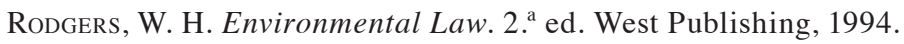

Rosanvallon, P. Counter-democracy Policy in the Age of Distrust. Cambridge, 2009.

Sands, P. Principles of International Environmental Law. 2. ed. Cambridge University Press, 2003.

Santamaría, J. Participación política y democracia directa. En Estudios de ciencia política y sociología: homenaje al profesor Carlos Ollero, Madrid: Editorial Nacional, 1972, 743-783.

Schlosberg, D. Defining Environmental Justice. Nueva York: Oxford University Press, 2007. 
Sмiтн, G. Deliberative Democracy and the Environment. Abingdon: Routledge, 2003.

Soто, F. El desafío de la participación. Estudio comparado sobre el referéndum y la iniciativa legislativa popular en América Latina y Europa. Santiago: Lom, 2013.

Sunstein, C. Riesgo y razón. Buenos Aires: Katz, 2006.

Tate, C. N. y Vallinder, T. (eds.). The Global Expansion of Judicial Power. New York University Press, 1995.

Teubner, G. Substantive and Reflexive Elements in Modern Law. En Law and Society Review. 1983, vol. 17, 239-285.

VAN BeKhoven, J. Public participation as a general principle in international environmental law: Its current. En National Taiwan University Law Review. 2016, vol. 11, 2, 219-271. 